\title{
Editorial
}

\section{Nonlinear Analysis: Algorithm, Convergence, and Applications 2014}

\author{
Yisheng Song, ${ }^{1,2}$ Rudong Chen, ${ }^{3}$ Guoyin $\mathrm{Li},{ }^{4}$ Changsen Yang, ${ }^{1}$ and Gaohang $\mathrm{Yu}^{5}$ \\ ${ }^{1}$ School of Mathematics and Information Science, Henan Normal University, Henan, China \\ ${ }^{2}$ Department of Applied Mathematics, The Hong Kong Polytechnic University, Hong Kong \\ ${ }^{3}$ Department of Mathematics, Tianjin Polytechnic University, Tianjin, China \\ ${ }^{4}$ Department of Applied Mathematics, University of New South Wales, Sydney, NSW 2052, Australia \\ ${ }^{5}$ Jiangxi Key Laboratory of Numerical Simulation Technology, School of Mathematics and Computer Sciences, \\ Gannan Normal University, Ganzhou, China \\ Correspondence should be addressed to Yisheng Song; songyisheng1@gmail.com
}

Received 14 September 2014; Accepted 14 September 2014; Published 22 December 2014

Copyright (C) 2014 Yisheng Song et al. This is an open access article distributed under the Creative Commons Attribution License, which permits unrestricted use, distribution, and reproduction in any medium, provided the original work is properly cited.

We are glad to achieve this special issue. This special issue was opened in late November of 2013 and closed in early February of 2014. There were 67 submissions in total, and 24 of them were accepted for publication after strict reviews, which gave important developments and applications in nonlinear analysis.

This special issue mainly concentrates on some latest developments and applications in nonlinear analysis. The topics of the accepted 22 articles cover various research fields of nonlinear analysis from theory to applications. Using techniques of nonlinear analysis, such as the fixed point theory and nonlinear spectral theory, as well as the iteration theory, many new methods and their analyzed techniques of convergence are presented for solving some nonlinear problems. Furthermore, some practical applications and numerical examples are given. These are our main aims of sponsoring this special issue.

The article by $\mathrm{H}$. Zegeye and N. Shahzad studied the strong convergence of Halpern iteration (1) for a finite family of right Bregman strongly nonexpansive mappings $\left\{T_{1}, T_{2}, \ldots, T_{N}\right\}$

$$
x_{n+1}=\alpha_{n} u+\left(1-\alpha_{n}\right) T x_{n}, \quad \text { where } T=T_{1} \circ T_{2} \circ \cdots \circ T_{N} .
$$

They obtained that the sequence $\left\{x_{n}\right\}$ strongly converges to a common fixed point of such a finite family in the framework of real reflexive Banach space, which applied to approximate a common zero of a finite family of maximal monotone mappings and a solution of a finite family of convex feasibility problems in real reflexive Banach spaces.

The article by $\mathrm{L}$. Shi et al. introduced an iterative algorithm to solve the multiple-sets split equality problem (MSSEP) in the framework of infinite-dimensional Hilbert spaces under some more mild conditions for the iterative coefficient. The multiple-sets split equality problem (MSSEP) is the following problem:

$$
\text { finding a point } x \in \bigcup_{i=1}^{N} C_{i}, \quad y \in \bigcup_{j=1}^{M} Q_{j} \quad \text { s.t. } A x=B y \text {, }
$$

where $\left\{C_{i}\right\}_{i=1}^{N},\left\{Q_{i}\right\}_{i=1}^{M}$ are two families of closed convex subsets of Hilbert spaces $H_{1}, H_{2}$, respectively, and $A: H_{1} \rightarrow H_{3}$, $B: H_{2} \rightarrow H_{3}$ are two bounded linear operators. It is obvious that MSSEP is called split equality problem (SEP) if $N=M=$ 1; MSSEP and SEP reduce to the well-known multiple-sets split feasibility problem (MSSFP) and split feasibility problem (SFP) if $B=I$.

The article by $\mathrm{H}$. He and S. Liu showed the strong convergence theorems of the CQ algorithm for $\mathrm{H}$-monotone operators in Hilbert spaces by hybrid method in the mathematical programming. 
The article by $\mathrm{Y}$. Wang and $\mathrm{H}$. Shi proved that a modified mixed Ishikawa iteration strongly converges to some common fixed point of two asymptotically quasipseudocontractive type non-self-mappings.

The article by L. Wei and R. Tan obtained strong convergence of iterative scheme with errors for finding some common zero of finite accretive operators and nonlinear elliptic systems and the relationship between zeros of $\mathrm{m}$ accretive operator and a class of nonlinear elliptic systems.

A kind of generalized complementarity problem (GCP) is defined by

$$
F(x) \geq 0, \quad G(x) \geq 0, \quad\langle F(x), G(x)\rangle \geq 0,
$$

where $F: R^{n} \rightarrow R^{n}, G: R^{n} \rightarrow R^{n}$ are two differentiable functions. The article by S.-Q. Du studied this class of generalized complementarity problem (GCP) and gave a generalized Newton method for finding some solution of such a problem. Furthermore, they showed the global convergence and superlinear local convergence results as well as some numerical results.

The article by H. Jiao et al. presented a novel optimization method for effectively solving the following nonconvex quadratically constrained quadratic programs (NQCQP) problem:

$$
\begin{array}{ll}
\min & G_{0}(x)=x^{T} A^{0} x+\left(d^{0}\right)^{T} x \\
\text { s.t. } & G_{i}(x)=x^{T} A^{i} x+\left(d^{i}\right)^{T} x \leq b_{i}, \quad i=1,2, \ldots, m \\
& x \in X^{0}=\left\{x \in R^{n}: l^{0} \leq x \leq u^{0}\right\},
\end{array}
$$

where $A^{i}$ are all symmetric matrices, $d^{0}, d^{i} \in R^{n}$, and $b_{i} \in R, i=1,2, \ldots, m$, and showed the convergence of the presented algorithm to the global optimum point of the NQCQP problem.

The article by L.-P. Yang et al. established a global optimization algorithm for solving globally the generalized nonlinear multiplicative programming (MP) with a nonconvex constraint set, which may be used to solve a general reverse convex programming problem and a series of linear programs that do not grow in size from iterations to iterations.

The article by J. Shen et al. introduced a cutting plane and level bundle method for minimizing nonsmooth nonconvex functions by combining cutting plane method with the ideas of proximity control and level constraint, which made a distinction between affine pieces that exhibit a convex or a concave behavior relative to the current iterate, and showed that the sequence defined by such a method converges to some kind of stationarity point.

The box-constrained optimization problem is given by

$$
\min _{x \in R^{n}} f(x), \quad l \leq x \leq u,
$$

where $f: R^{n} \rightarrow R$ is continuously differentiable and $l, u \in$ $R^{n}$, with $l<u$. In the paper by Q. Wang and Y. Che, a practical algorithm for solving large-scale box-constrained optimization problems is developed, analyzed, and tested. Under some appropriate conditions, they showed the global convergence of the algorithm and gave the numerical experiments and comparisons by using some box-constrained problems.

The article by Y.-H. Ren analyzed the second-order multiplier iteration based on a class of nonlinear Lagrangian algorithm for solving nonlinear programming problems with inequality constraints.

The article by Y. Qiu et al. studied a nonmonotone adaptive Barzilai-Borwein gradient algorithm for $l_{1}$-norm minimization problems arising from compressed sensing and established its global convergence result under some suitable conditions.

The article by X.-P. Hou et al. presented a global optimization algorithm for solving the signomial geometric programming (SGP) problem. They first convert the initial nonconvex programming problem (SGP) into an equivalent monotonic optimization problem and proved the convergence of such an algorithm to the global minimum of the SGP by means of the subsequent solutions of a series of relaxation linear programming problems.

The article by J. Yuan and C. Wang considered the Furutatype inequalities and their applications and obtained a unified satellite theorem of grand Furuta inequality and some Furutatype inequalities under $A \geq B \geq 0$ via Loewner-Heinz inequality.

The article by H. Li and T. Ma investigated the boundedness and compactness of generalized composition operators $C_{\varphi}^{g}$ from $\left(\mathbf{B}_{\mu}\left(\mathbf{B}_{\mu, 0}\right)\right)$ spaces to spaces $Q_{K, \omega}(p, q)$. The other article by the same authors studied an iterative process which converges strongly to a zero of a finite sum of monotone mappings.

The article by F. Zuo and J. Shen provided some conditions for $2 \times 2$ operator matrices whose diagonal entries are $M$-hyponormal operators to be subscalar and showed the Weyl-type theorem of such operator matrices

The article by X. Li and F. Gao considered the tensor products for class $A(n)$ operators (an operator $T \in B(H)$ is called a class $A(n)$ operator if $\left.\left|T^{1+n}\right|^{2 /(1+n)} \geq|T|^{2}\right)$ and gave a necessary and sufficient condition for $T \otimes S$ to be a class $A(n)$ operator when $T$ and $S$ are both nonzero operators.

The article by $\mathrm{H}$. Wang et al. considered the Cauchy problem for a fifth-order equation and proved its locally wellposed for the initial data in the Sobolev space $H^{s}(R)$ with $s \geq 1 / 4$. Moreover, they also established the ill-posedness for the initial data in $H^{s}(R)$ with $s<1 / 4$.

The article by $\mathrm{C}$. Li et al. used the principal component analysis method to achieve dimensionality and correlation of input variables and obtained the three major factors affecting membrane fouling most obviously: MLSS, total resistance, and operating pressure, and applied genetic algorithm to optimize the initial weights and the threshold of BP neural network and established the membrane fouling prediction model based on GA-BP network.

$$
\begin{array}{r}
\text { Yisheng Song } \\
\text { Rudong Chen } \\
\text { Guoyin Li } \\
\text { Changsen Yang } \\
\text { Gaohang Yu }
\end{array}
$$




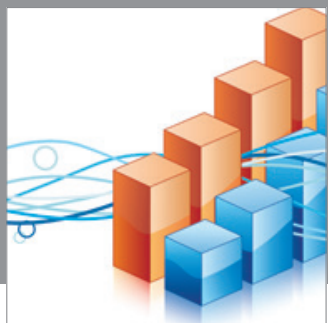

Advances in

Operations Research

mansans

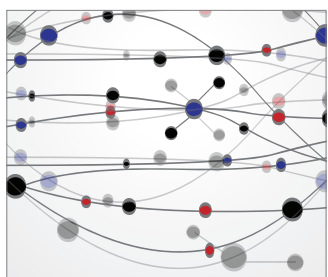

The Scientific World Journal
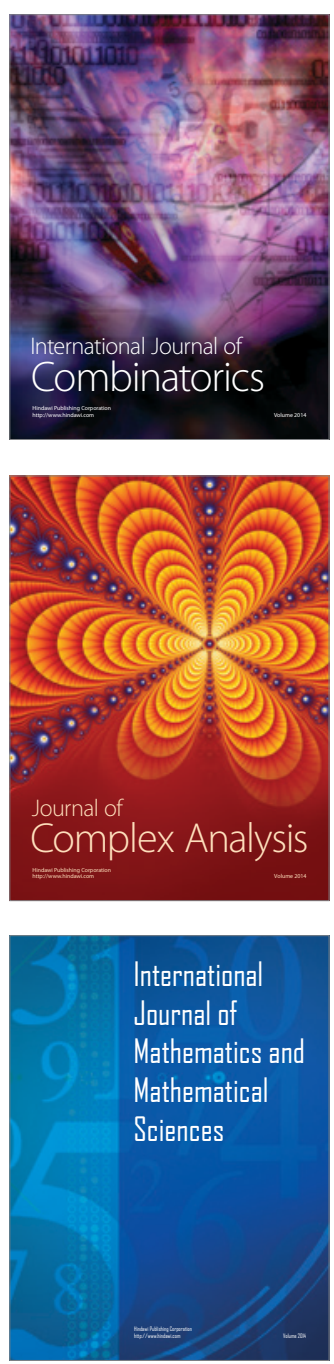
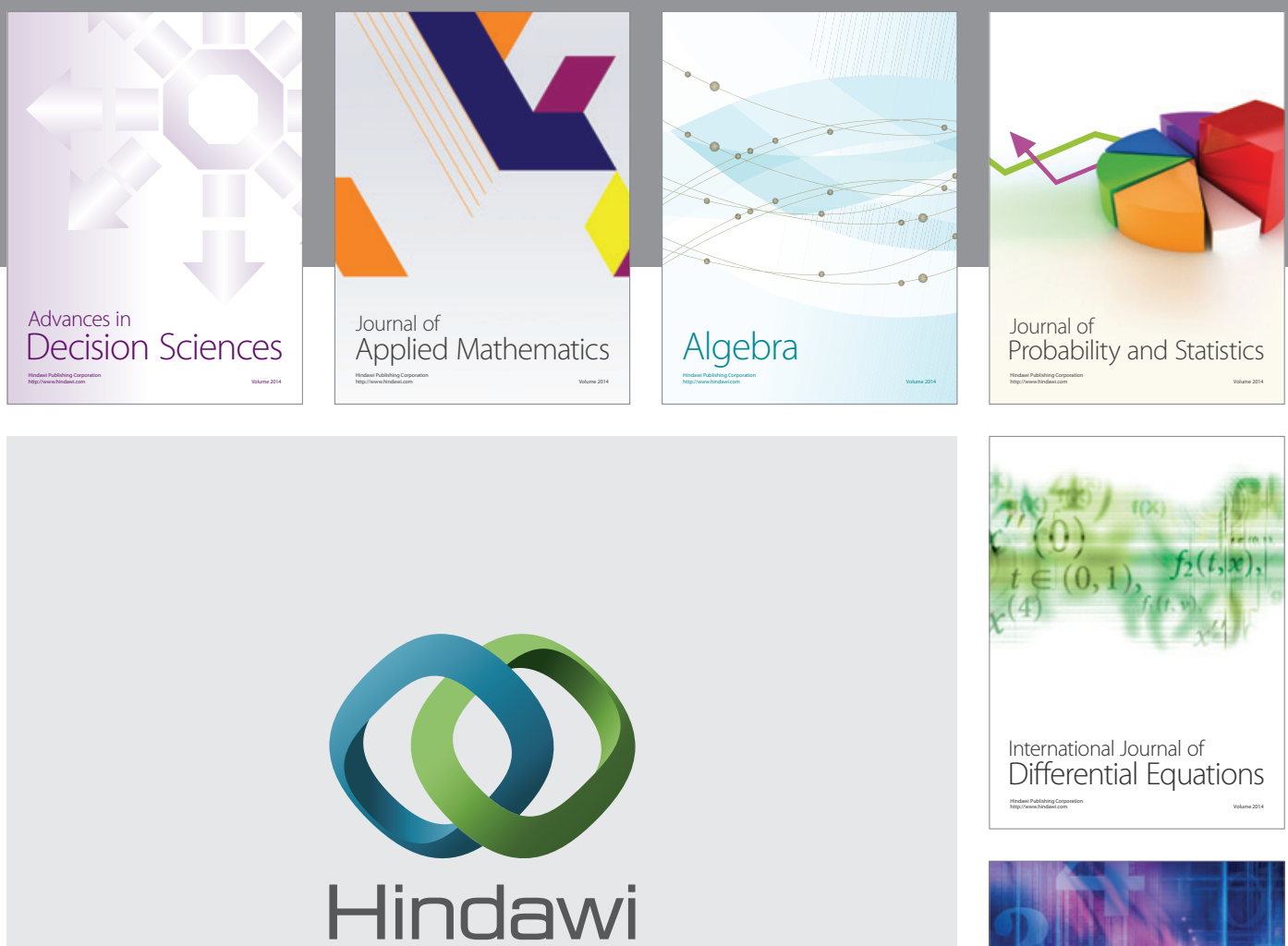

Submit your manuscripts at http://www.hindawi.com
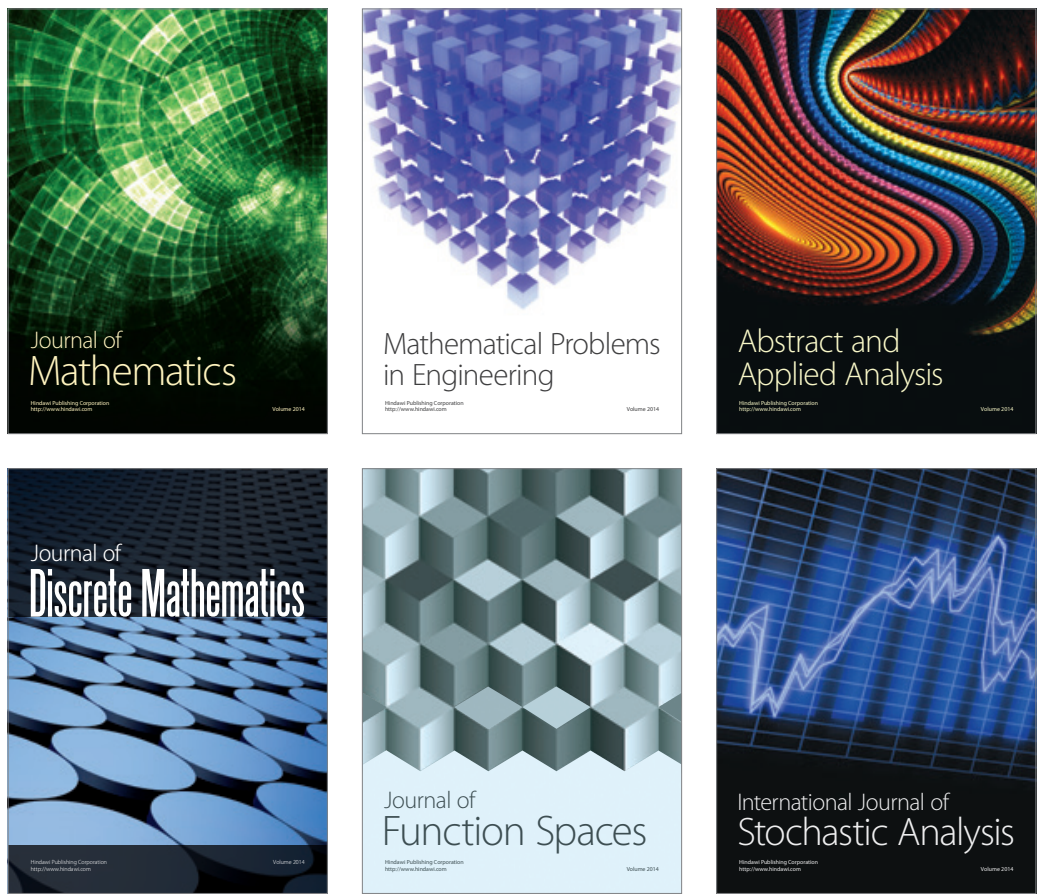

Journal of

Function Spaces

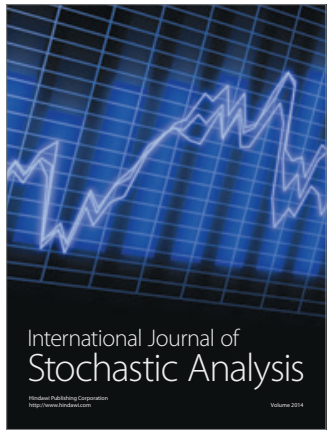

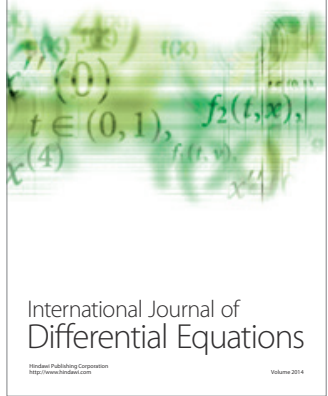
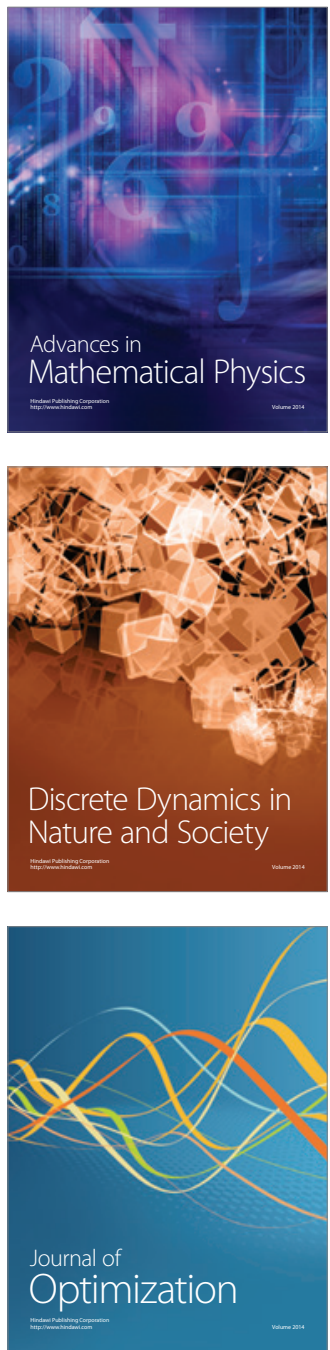